\title{
Photovoltaic modules based on automatic biochemical analyzer colorimetric system optimization design
}

\author{
Hu Wang ${ }^{2, \mathrm{~b}}$, Zhi-Gang $\mathrm{Hu}^{1, \mathrm{a}}$, Zhe $\mathrm{Du}^{1}$ and Zhen-Dong Shang ${ }^{2}$ \\ ${ }^{l}$ Medical Technology and Engineering, Henan University of Science and Technology . \\ Luoyang, Henan, China \\ ${ }^{2}$ Mechanical and Electrical Engineering, Henan University of Science and Technology. \\ Luoyang, Henan, China \\ E-mail: ${ }^{a}$ hu.robert@163.com, ${ }^{b} 1139360593 @ q q . c o m$
}

\begin{abstract}
In this paper, 8-channel photovoltaic modules was made up of the spectral device, monochromator and amplifier circuit. Combining with the spectroscopic techniques, an automatic biochemical analyzer colorimetric system was designed. The $12 \mathrm{~V}$ halogen lamp which adds stabilizer and a cooling fan was used as light source. The band full width at half maximum of $10 \mathrm{~nm}$ filter was selected as spectroscopic device. The PIN13DSB photoelectric diode was chosen as photoelectric converter. The amplifier circuit was designed by using Photovoltaic mode. Compared with traditional biochemical analyzer, the experiment shows that this design method can reduce the color dark noise of the system, and improve the accuracy of colorimetric system testing.

Keywords: Photovoltaic Modules; Analysis; Optical Design; Circuit Design.
\end{abstract}

\section{Introduction}

Automatic biochemical analyzer is an instrument that adopts photoelectric colorimeter principle to measure a particular chemical composition of blood or body fluids. At present, the light path of large-scale biochemical analyzer at home and abroad commonly used grating structure, such as BS-480 invented by Shenzhen Mindray company. It uses holographic concave flat field grating rearspectrophotometer road construction. Although it boasts a high precision, it is expensive and difficult to install and debug, which makes it not appropriate to be used in small and medium biochemical analyzer. The mainstream optical structure used on SMES biochemical analyzer still adopts conventional filter structure. The main problems of interference filter optical system used in most domestic small and medium biochemistry analyzer are as follows:1)regional differences lead to the vulnerability of optical system to interference, which affects detection accuracy. 2) changes in temperature and humidity facilitates 
the growth of visible mold on the filter. 3)Using the lamp for long results in insufficient brightness, and it is difficult to replace [1].

Based on the above issues, this paper presents a sealed photovoltaic module consisting of the monochromator, detector combinations and the photoelectric conversion section to optimize the structure of the optical system and to resolve the vulnerability of the optical system to interference and difficulty in lamp replacement. Photovoltaic module in a closed metal shield box isolates the filters from the effect of temperature and humidity changes and improves the detecting speed and accuracy of the colorimetric system in the meantime.

\section{The Functioning Principle of Biochemical Analyzer}

At present, the colorimetric principle commonly adopts biochemical analyzer Lange-Beer law: when a bundle of parallel monochromatic light transmits through uniformly colored solution, since the solution absorbs part of the light, the transmitted light intensity weakens. The greater the concentration, the thicker liquid layer, the stronger the incident light, transmitted light intensity decreases more significantly [2]. The relationship between the incident light and the transmitted light is presented in Eq.(1):

$$
\mathrm{A}=-\log \left(\mathrm{I}_{\mathrm{t}} / \mathrm{I}_{\mathrm{o}}\right)=\mathrm{K} \cdot \mathrm{C} \cdot L
$$

In the formula above, Io stands for the intensity of the incident light; $\mathrm{K}$ stands for the molar extinction coefficient; $\mathrm{C}$ stands for the concentration; $\mathrm{L}$ stands for the thickness of the liquid layer; and A stands for the absorbance of the solution. Supposing there are two colored solutions, one is the calibration solution whose concentration is known, and the other is the test solution. The absorbance of calibration solution is represented by $A_{S}$, and the absorbance of test solution is represented by $A_{U}$. Both solutions satisfy Eq. (1):

$$
\begin{aligned}
& \mathrm{A}_{\mathrm{S}}=\mathrm{K}_{\mathrm{S}} \cdot C_{S} \cdot L_{S} \\
& \mathrm{~A}_{\mathrm{U}}=\mathrm{K}_{\mathrm{U}} \cdot C_{U} \cdot L_{U}
\end{aligned}
$$

It is assumed equal in the thickness of the liquid layer of the two solutions: $\mathrm{L}_{\mathrm{S}}$ approximately equals $\mathrm{L}_{\mathrm{U}}$. If the temperature, the substance of the two solutions, and the determination of the selected wavelength of monochromatic light are the same, or $\mathrm{K}_{\mathrm{S}}$ approximately equals $\mathrm{K}_{\mathrm{U}}$, two simultaneous formula can be reached as follows:

$$
\frac{A_{S}}{A_{U}}=\frac{K_{S} \cdot C_{S} \cdot L_{S}}{K_{U} \cdot C_{U} \cdot L_{U}} \Rightarrow C_{U}=\frac{A_{U}}{A_{S}} \cdot C_{S}
$$


Eq. (2) shows that absorbance measured in the test solution can be found and the concentration of the test solution can reflect the health of the patient. Automatic biochemical analyzer colorimetric system is an optical signal through the colored solution within the cuvette; the solution absorbs part of the light energy that is then converted into a corresponding electric signal by the photodetector through the signal conditioning circuit simulation / digital (A / D) conversion and sampled, MCU (microcontroller Unit; MCU) used for data processing. In the meantime, MCU control motor-driven sample tray 8 signals to ensure that sampling and sample cleaning are carried out simultaneously.

\section{The Optical Portion of Colorimetric System}

Automatic biochemical analyzer has two spectral colorimetric method: spectrophotometry before and after splitting, but due to the low speed of testing before splitting, multi-wavelength cannot be tested, so this method is not considered in this article. After spectroscopic techniques can be a photodiode array, planar array charge-coupled device (Charge-coupled Device, CCD) and a multi-wavelength tester because it is to go through the cuvette and then through monochromator splitting. As technology is developing, after spectroscopic techniques has become more mature with simpler structures and acceptable costs for SMEs. Therefore, after spectrophotometric method is selected.

After splitting with technology, two designs are listed in the article (see Fig. 1): (a) represents an optical system solutions for the traditional biochemical analyzer of a particular model; (b) represents the design adopted in the article, including optimization of the optical structure, redesigned amplifier circuit, the combination of the optical portion of the photoelectric conversion part to the photovoltaic module.
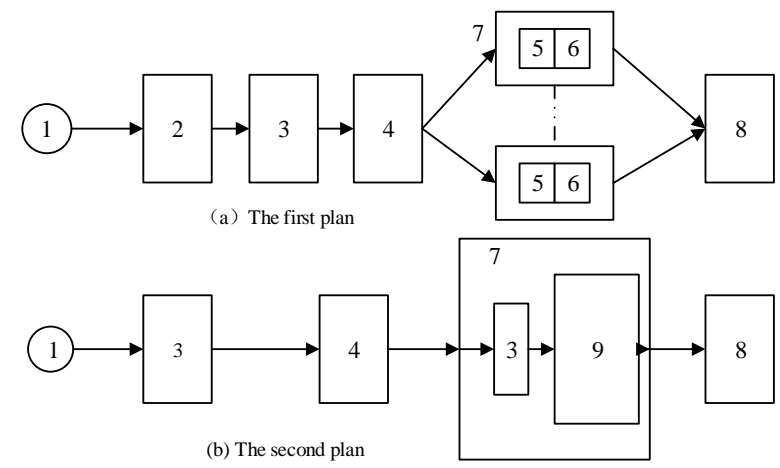

Fig. 1 Two optical systems design

1- light source; 2- condenser lens group; 3- fiber coupler; 4- cuvette; 5- photodetector; 6- amplifier; 7- metal shield box; 8- stage signal processing system; 9-8 road signal acquisition module; 
In the design shown in Figure1.(a), by focusing light into the fiber-optic, the fiber-optic bundle 8 into the optical path into the same sealed preamplifier version 8. The design shown in Fig.1.(b), the one adopted in this paper, simplifies the light source system. $12 \mathrm{~V}$ halogen light source emits light into the fiber-optic, through the lens module and arriving cuvette, and then into the module, spectral light sources, filters, photoelectric conversion and signal amplification all in a metal box.

The optical portion of colorimetric system consists of a light source, spectrometer, cuvettes and other components. Wherein, the light source needs to be transmitted $200 \mathrm{~nm}-1000 \mathrm{~nm}$ continuous spectrum ${ }^{[3]}$. You need to issue a certain intensity of UV light because the $340 \mathrm{~nm}$ UV light is often used biochemical analyzer test items. Considering quartz halogen lamps meet the above requirements, and that the stability of the instrument meets alanine aminotransferase (Alanine aminotransferase, ALT) in the test, amylase (Amylase, AMY) and other sources of power attenuation requirements, $12 \mathrm{~V}$ halogen lamp is chosen and installation of a voltage stabilizer. Since the light source itself generates heat very seriously while emitting, it will reduce the service life of the light source. Therefore a cooling fan is used for for cooling of halogen lamps.

\section{Optical Module}

This paper presents part of the photoelectric conversion portion of the light path making up a combination of 8-channel photovoltaic modules, to simplify the structure colorimetric system, to facilitate the production, installation and maintenance. Optical spectrometer module consists of splitting means, a photodetector and amplifying circuit.

The light source emits broad band polychromatic light. In the actual measurement process, a narrow band or monochromatic light needs adopting. The use of narrow band energy enables measurement to be conducted in the reagents within the cuvette at the wavelength of maximum absorption to improve measurement accuracy and sensitivity. Therefore, a reasonable splitting method can improve color accuracy from the source. Typical raster splitting methods are grating, filters and prisms .

Compared with grating and a prism, the filter grating is less expensive and has high sensitivity and high light transmittance rate in chemiluminescent detection. The main factor that affects the characteristics of the optical filter is band half-width. In accordance with the relationship between the optical 
wavelength and the band half-width, the filter with half-width of $10 \mathrm{~nm}$ band is selected.

The optical signal is difficult to measure quantitatively, and therefore requires that photodetector convert the optical signal to an electric signal. Common photodetectors include light battery, photocell and the photomultiplier tubes. Biochemical analyzer with a photoelectric detector must meet the requirement of high sensitivity, fast response, and electrical signal must be easy for detection and amplification. Moreover, low noise is also a prerequisite. This article selects PIN-13DSB photodiode, a blue-enhanced silicon photodiode sensitive to the wavelength range of $320-1200 \mathrm{~nm}$, which enjoys a low bias capacitance, fast response, stable, low noise, no external bias at work voltage and so on.

The electric signal converted by photodetector is very weak. For the same light source, monochromatic lights obtained through monochromator are different in intensity ${ }^{[4]}$. Therefore, we need to design a practical preamplifier to convert the current signal sensed by front stage photodetector to a voltage signal to a certain level of amplifier unit for subsequent processing. A photodiode detector has two modes: a photovoltaic mode and the light guide mode.

Compared with the photoconductive mode, the photodiode in photovoltaic mode is at a zero-bias state, where there is no dark current, low noise, good linearity, suitable for detecting faint light signals. Because the electric signal converted by photodetector is very weak and vulnerable to noise outside, reducing noise is essential for designing amplifier. Reducing the noise effectively means limiting the noise bandwidth by decoupling phase compensation. The final amplifier circuit is shown in Fig. 2:

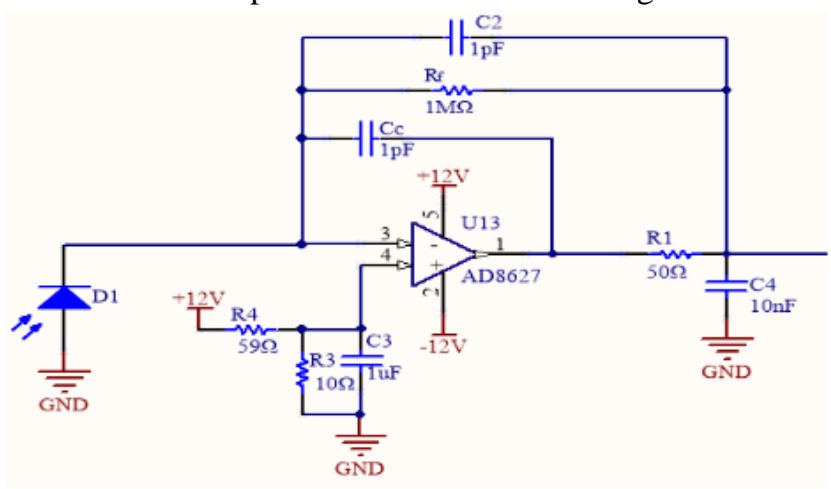

Fig. 2 Preamplifier circuit 
In the circuit, signal bandwidth can be limited by increasing the $\mathrm{C} 2$, in order to achieve the purpose of filtering, but the noise bandwidth cannot be limited by increasing the $\mathrm{C}^{[5]}$. Therefore an $\mathrm{RC}$ lowpass filter circuit is added between the output amplifier and the output of the detection circuit to filter out the amplified noise and noise from amplifier itself. R4 and R3 are used for improving the inverting input current to guarantee that the amplifier functions within a certain linear range of areas. Phase compensation capacitor CC is to compensate for delay caused by the filter circuit. A capacitor C2 is used to compensate for phase lag caused by photodiode junction capacitance $\mathrm{CD}$ to suppress noise gain peaking. Usually make R1 approximately equals Ro, Ro is the equivalent output resistance of the amplifier, generally based on the value of experience. It is generally assumed that

$$
\mathrm{R}_{\mathrm{o}} \approx 50 \Omega, C_{C} \approx C_{2}, \mathrm{C}_{4}=\frac{\mathrm{R}_{\mathrm{f}}}{\mathrm{R}_{\mathrm{O}}+\mathrm{R}_{1}} \mathrm{C}_{2}
$$

\section{Experimental Research and Analysis}

The electrical signal converted from the optical signal in preamplifier circuit are relatively weak because the feedback resistor of the amplifier should not be too large. Otherwise it will cause the self-excited oscillation, and anti-interference ability deteriorates. Therefore two amplifier need to be added in the subsequent two amplifier circuits. D conversion section uses MAX1300 chip that is 16-bit successive approximation ADC with 115KSPS throughput. By programming, the paper intends to analyse the noise and the experimental accuracy of the two schemes.

\subsection{Analysis of the dark noise of experimental colorimetric systems}

In order to prove the merits and disadvantages of the two approaches mentioned above, the two optical devices are disposed under the same experimental conditions, to 0 (no light) as a reference experiment. In theory, the photoelectric detector are placed in a sealed metal box, with external electromagnetic interference shielded and the amplifier output terminals adjusted to zero. After the experiment, the measured output of the two designs are shown in Fig. 3: 


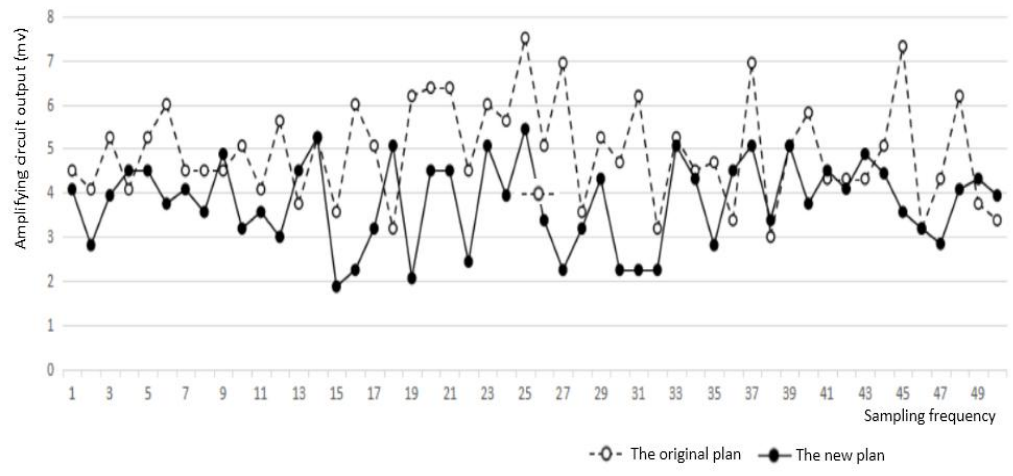

Fig. 3 Comparison among 50 experimental data without illumination

From the results found herein, the design of the program is more stable. The measured result is closer to 0 than conventional approaches. The average and variance of the data are shown in Fig. 4.

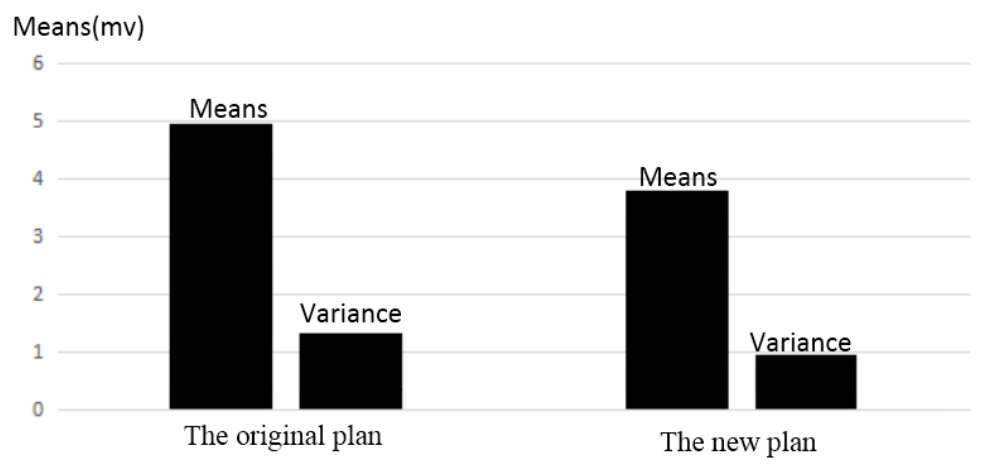

Fig. 4 Comparison between the two approaches in average and variance

Experimental results show that the photovoltaic module data is smaller than the original design in the absence of light, thus being more stable. The average voltage V0 of the two programs were 4.92825 and 3.79045 respectively. The new program enjoys a smaller average than the original program. It indicates that the new scheme reduces the dark noise of the colorimetric system and improves the performance of colorimetric system. Because it is an 8-channel simultaneous sampling, colorimetric system is undoubtedly faster than the original design. But the output of two optical systems on the condition of no illumination is not zero, which is due to the influence of the dark current and the following circuit. 


\subsection{Calibration parameter analytical experiment of colorimetric system}

The end point assay reactivity was adopted in the experiment, and the testing process is presented as follows:

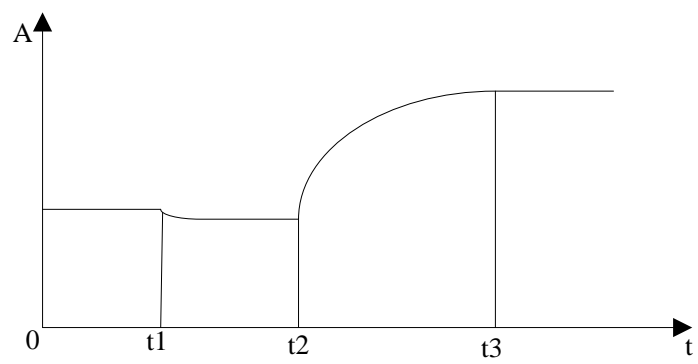

Fig. 5 The chart of single-wavelength, single-agent formula Endpoint test

As is shown in Figure5, at the time of $\mathrm{t} 1$ the reagents were added; at the time of $\mathrm{t} 2$ the sample was added; the absorbance was measured after the reaction solution was stirred; at the time of $\mathrm{t} 3$ the reaction came to an end; $\mathrm{t} 2-\mathrm{t} 3$ is a period of measurement.The formula of the reaction is:

$$
R=A_{t 3}-A_{0}
$$

At3 represents the absorbance at the time of $\mathrm{t} 3$; A0 represents singlereagent blank absorbance.

Total Protein reagent with a dominant wavelength of $546 \mathrm{~nm}$ was selected as the standard to test the accuracy of colorimetric system. The calibration mode is single-point linear calibration. The concentration of the reagent is calculated as follows:

$$
C=\frac{R}{K}
$$

$\mathrm{C}$ is the concentration of the reagent; $\mathrm{R}$ is the reagent of the reaction; $\mathrm{K}$ is the calibration parameters.

According to Eq.4, the accuracy of the concentration of the reagent depends on the accuracy of the calibration parameters.

To determine the standard solution, 10 groups of total protein reagents of different concentrations were selected. When the blank test of the reagents was carried out, the magnification degree of the amplifier circuit of the two programs was adjusted so that the data of measured absorbance were equal and to ensure that the blank absorbance, A0 of a single reagent was the same. The measured degree of response are shown in the following Fig. 6: 


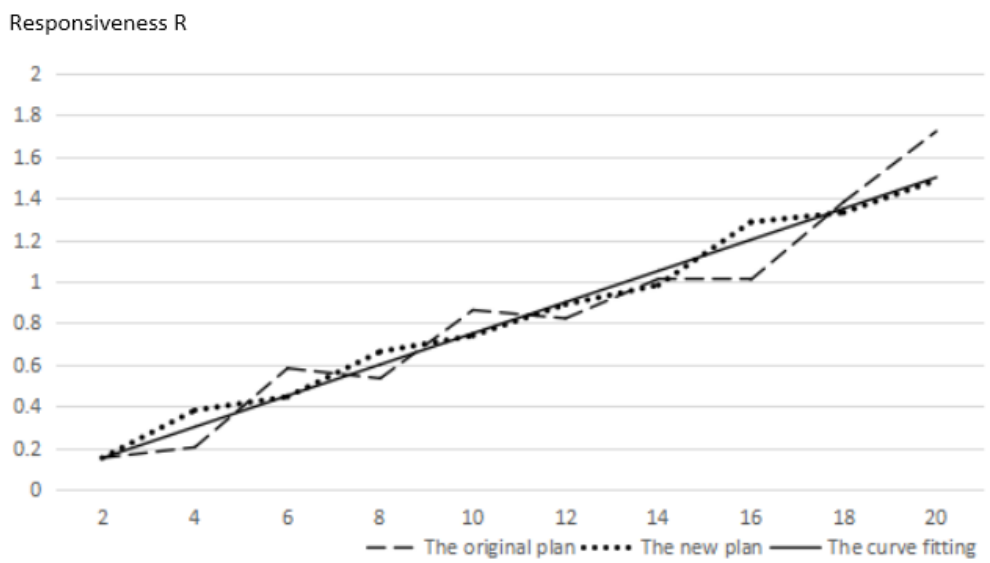

Fig. 6 responsiveness - concentration curve of the two schemes

Least squares curve fitting are conducted on the data of the two schemes. The fitting curve equation of the both programs approximate the following equation: $R=0.075 C$

Comparison of two schemes with the data curve fitting variance:

$$
\left\{\begin{array}{l}
S^{2}=\frac{1}{n}\left[\sum\left(R_{i}-Y_{i}\right)^{2}\right] \\
Y_{i}=0.075 i, i=2,4,6 \ldots 20
\end{array}\right.
$$

By Eq. (5), it is calculated as:

The variance of the original plan: $S_{1}{ }^{2}=0.111222$

The variance of the new plan: $S_{2}{ }^{2}=0.012226$

The variance of the new progam is smaller than that of the original plan, so the fluctuations of the new scheme are smaller. In determining the actual calibration parameters, the concentration of a solution is determined as the calibration parameter $\mathrm{K}$; according to the Eq.4 the detection of the reaction can be done after the sample is added; subsequently the concentration of sample reagent mixture can be calculated. By the comparison of the variance of the two schemes with respect to that of the fitted curve, we can conclude that new data distribution scheme fits closer to the vicinity of the curve, and that the new program is more accurate in determining the calibration parameters. Since the accuracy of the calibration parameters directly affect detection accuracy of the results of the colorimetric system. The new program is more accurate in detection. 


\section{Conclusion}

Based on the photovoltaic module, the photoelectric detection system of the automatic biochemical analyzer was designed.

(1) Using rear-spectroscopic techniques can simplify the structure and be cost-effective. Combining the splitting means, monochromator and amplifier into an 8-channel photovoltaic modules can greatly improve the reliability and response speed of the system.

(2) $12 \mathrm{~V}$ halogen light source and the installation of the regulator and cooling fan meet the requirements of system parameters; spectral selection means select the band with the half width of $10 \mathrm{~nm}$ filter, select Select PIN13DSB photoelectric conversion photodiode, and adopt photovoltaic mode amplifier circuit design.

(3) Comparison with conventional biochemical analyzer experiments show that the design method reduces the dark noise of the colorimetric system and improves the detection accuracy of the colorimetric system.

\section{Acknowledgments}

The cooperation projects of enterprises, universities and research institutes in Henan (2015HNCXY003); Henan Science and Technology research programs $(122,102,310,276)$.

\section{References}

1. Guo Youcheng.The maintenance and repair of the optical path of HitaChi 7020 automatic biochemical analyzer[J]. Chinese Medical Equipment Journal.2014,35(5).

2. Chen Guan,Xiao Zexin.Research and design of biochemical analyzer optical system[J].Optical Technique,2006,32(z1):374-379.

3. Liu Yu,Wang Ling.Exploration of Biochemical Analyzer Source Power Attenuation Indications[J],China Medical Devices,2014,(4):30-32.

4. Wan Hanwei,Long Wei .Design and application of a high precision spectroscopy signal acquisition system[J],Journal of Nanchang University(Natural Science) ,2013,37(1):26-29.

5. Jia Zhenan,Zheng Delin.Demodulation Electric Circuit of Optical Fiber Grating Weak Signal Detection[J].Instrument Technique and Sensor,2012,(5):79-81. 